\title{
Allocation, not male resistance, increases male frequency during epidemics: a case study in facultatively sexual hosts
}

\author{
Jessica L. Hite, ${ }^{1,5,8}$ Rachel M. Penczykowski, ${ }^{2,6}$ Marta S. Shocket,,${ }^{1,7}$ Katherine A. Griebel, ${ }^{1}$ \\ Alexander T. Strauss, ${ }^{1}$ Meghan A. Duffy, ${ }^{3}$ Carla E. Cáceres, ${ }^{4}$ and Spencer R. Hall ${ }^{1}$ \\ ${ }^{1}$ Department of Biology, Indiana University, Bloomington, Indiana 47405 USA \\ ${ }^{2}$ School of Biology, Georgia Institute of Technology, Atlanta, Georgia 30332 USA \\ ${ }^{3}$ Department of Ecology and Evolutionary Biology, University of Michigan, Ann Arbor, Michigan 48109 USA \\ ${ }^{4}$ School of Integrative Biology, University of Illinois at Urbana-Champaign, Urbana, Illinois 61801 USA
}

\begin{abstract}
Why do natural populations vary in the frequency of sexual reproduction? Virulent parasites may help explain why sex is favored during disease epidemics. To illustrate, we show a higher frequency of males and sexually produced offspring in natural populations of a facultative parthenogenetic host during fungal epidemics. In a multi-year survey of 32 lakes, the frequency of males (an index of sex) was higher in populations of zooplankton hosts with larger epidemics. A lake mesocosm experiment established causality: experimental epidemics produced a higher frequency of males relative to disease-free controls. One common explanation for such a pattern involves Red Queen (RQ) dynamics. However, this particular system lacks key genetic specificity mechanisms required for the RQ, so we evaluated two other hypotheses. First, individual females, when stressed by infection, could increase production of male offspring vs. female offspring (a tenant of the "Abandon Ship" theory). Data from a life table experiment supports this mechanism. Second, higher male frequency during epidemics could reflect a purely demographic process (illustrated with a demographic model): males could resist infection more than females (via size-based differences in resistance and mortality). However, we found no support for this resistance mechanism. A size-based model of resistance, parameterized with data, revealed why: higher male susceptibility negated the lower exposure (a size-based advantage) of males. These results suggest that parasite-mediated increases in allocation to sex by individual females, rather than male resistance, increased the frequency of sex during larger disease epidemics.
\end{abstract}

Key words: Daphnia; parasite; parthenogenic; resistance; sex allocation; sex-specific infection.

\section{INTRODUCTION}

Natural populations exhibit substantial variation in the frequency of sexual reproduction (O'Connell and Eckert 2001, Tessier and Cáceres 2004, Jokela et al. 2009). Given the myriad costs associated with sexual reproduction (Maynard Smith 1978, Bell 1982), it remains challenging to identify general mechanisms driving population-level variation in sex (reviewed by Hartfield and Keightley 2012). The Red-Queen hypothesis poses that parasites can promote more sex (Hamilton et al. 1990, Decaestecker et al. 2003, Jokela et al. 2009). Here, parasites can increase the frequency of sexually reproducing hosts via negative frequency-dependent

Manuscript received 18 March 2017; revised 23 June 2017; accepted 18 July 2017. Corresponding Editor: Auston Marm Kilpatrick.

${ }^{5}$ Present address: School of Biological Sciences, University of Nebraska, Lincoln, Nebraska 68588 USA.

6 Present address: Department of Biology, Washington University, St. Louis, MO 63130 USA.

7 Present address: Department of Biology, Stanford University, Stanford, California 94305 USA.

${ }^{8}$ E-mail: jhite2@unl.edu selection, i.e., by sometimes granting sexual offspring fitness advantages over asexual ones (via host-parasite coevolution; Bell 1982). However, the Red-Queen does not apply to all host-parasite systems; mechanistically, it requires assumptions about specificity of infection genetics between the host and parasite. Numerous host-parasite systems lack these natural history ingredients (e.g., Clay and Kover 1996, Stelzer 2015, and the focal system here). Hence, critics argue that the Red Queen remains too restrictive to generally drive population-level variation in sex (Salathé et al. 2008, Otto 2009, Stelzer 2015).

An alternative, "Abandon Ship" hypothesis links stress to sex (Hadany and Otto 2009, Mostowy and Engelstädter 2012). Stressors including drought, starvation, crowding, and predators can drive increased allocation to sex in a diverse array of organisms including facultative parthenogens (e.g., Daphnia [Cáceres and Tessier 2004], moths [Kumpulainen et al. 2004]) and strictly sexual species (e.g., plants [Griffiths and Bonser 2013]); (for more comprehensive lists see Hadany and Otto [2009] and Mostowy and Engelstädter [2012]). In the broadest sense, the Abandon Ship hypothesis posits that individual females in the poorest condition increase 
allocation to sex to escape eminent threats via dormancy or production of genetically diverse offspring (Hadany and Otto 2009). Hence, sex serves as a bet-hedging strategy governed by an individual's fitness prospects in the local environment. If variation in sex depends on the severity of stress, changes in environmental stressors could explain why natural populations vary in the frequency of sexual reproduction.

Here, we examine the ability of parasite-induced stress to drive allocation to sex in their hosts. While several studies have shown that parasites often increase outcrossing (e.g., Lucht et al. 2002, Kovalchuk et al. 2003), few studies have linked parasite-induced stress to sex-allocation decisions by individual hosts (but see Duncan et al. 2009, 2006). This conceptual gap is quite surprising. Parasites are ubiquitous, often virulently exert physiological stress on hosts, and create spatiotemporal variation in infection-induced stress. Therefore, parasites likely serve as a key stressor catalyzing sex investment in non-Red Queen systems.

However, a positive correlation between epidemics and sex could also emerge through an alternative, nonallocation mechanism. If males resist infection more than females, male frequency could increase during disease epidemics through demography, not via allocation decisions by individual females. In other words, male frequency could increase if females suffer greater parasiteinduced mortality. Such asymmetric mortality could leave behind more resistant males. To date, this hypothesis has remained mathematically and conceptually underdeveloped, despite that males often resist infection (e.g., in Lyme disease [Jarefors et al. 2006], schistosomiasis [Remoue et al. 2001], toxoplasma [Walker et al. 1997], and babesia [Aguilar-Delfin et al. 2001]). Higher male resistance can arise through size-based, behavioral, or immunological traits that govern exposure or susceptibility to parasites (e.g., Moore and Wilson 2002, Perkins et al. 2003, Cousineau and Alizon 2014). Hence, male resistance, in its purely demographic form modeled here, could provide an underappreciated, yet important, alternative to more typical hypotheses attributing benefits of sex during epidemics.

We evaluate these two mechanisms (allocation vs. male resistance) using a facultatively sexual host (Daphnia dentifera; hereafter, "hosts") and its virulent fungal parasite (Metschnikowia bicuspidata; hereafter, "fungus" (Ebert 2005, Hall et al. 2009). In this system, the frequency of males provides an index of sexual reproduction and investment of hosts. We can use this system to link individual-level traits (e.g., allocation to male offspring by adult females, or male vs. female resistance) to population-level variation in sexual reproduction during natural and experimental epidemics. We combine multiple modes of inference to eventually confirm a parasitedriven allocation to sex mechanism. First, we illustrate a focal pattern: we see higher male frequency during larger fungal epidemics among natural lakes. We confirmed that parasites can cause higher male frequency using a mesocosm experiment deployed in a lake during the epidemic season (this experiment helps rule out other covarying factors among lakes that could drive the field correlation). Then, a life table assay showed higher allocation to males by infected females. Finally, we ruled out a more complicated male resistance hypothesis, despite field and mesocosm data that seemed to support it (at first glance at least). We used a demographic model to clarify conditions linking male resistance to higher male frequency during epidemics. However, a size-based resistance model revealed that males do not resist infection more than females (despite a priori, size-based expectations). Together, our results suggest that parasiteincreased allocation to sex, rather than male resistance, drove this male-frequency-epidemic-size pattern.

\section{Natural History of the Host-Parasite System}

The focal hosts are key consumers in food webs in north temperate freshwater lakes. These facultative parthenogenetic hosts produce asexual broods of females throughout most of the year (Fig. 1). However, environmental stress that signals winter's onset (e.g., decreasing water temperatures and daylight) induces some females to increase allocation to sex (these females can then alternate between sexual and asexual reproduction and can produce mixed broods with male and female offspring). Sexual reproduction involves (1) the production of males and (2) eggs inside a durable case,

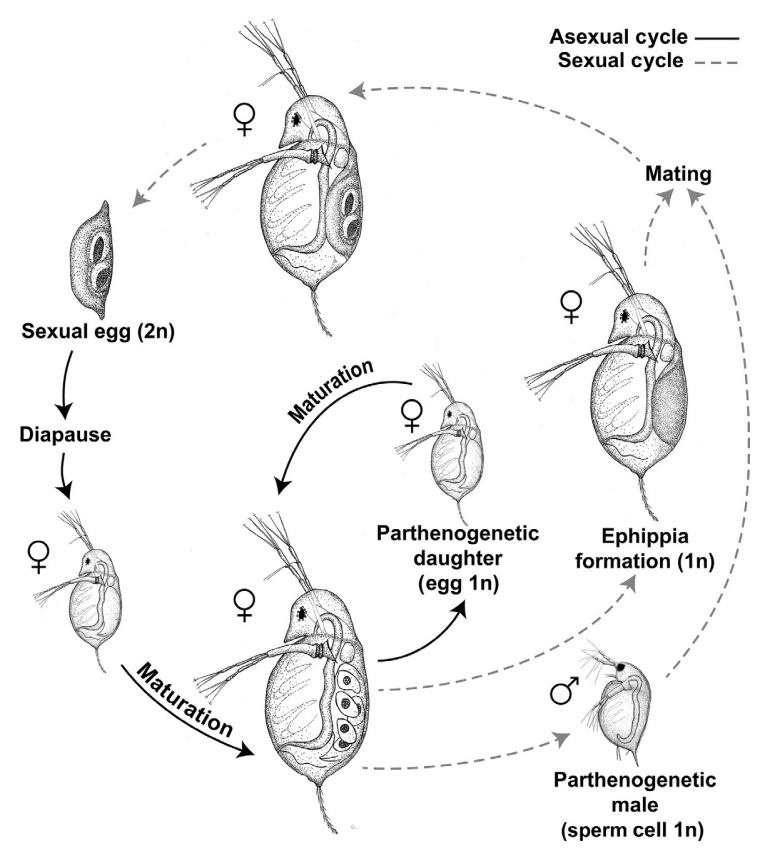

FIG. 1. Life cycle of the host, Daphnia dentifera. Solid lines depict the asexual parthenogenetic phase. Dashed lines depict the sexual phase. Numbers in parentheses reflect the ploidy of the gametes produced by different stages. Note the smaller size of adult males relative to adult females. Illustration by Julia Ferguson. 
called an ephippium. Males then fertilize these eggs, creating genetically diverse resting eggs that can survive winter inside the protective and durable ephippia (Ebert 2005; Fig. 1). This temporal sequence often produces a positive relationship between the frequency of males and ephippia-carrying females (Tessier and Cáceres 2004). Thus, the frequency of males indexes the host's investment in sexual reproduction.

The decline of conditions from fall to winter also coincides with peak infection by the fungal parasite. Before ultimately killing the host, the parasite fills the host's body cavity with spores; upon host death, spores are released into the environment where hosts inadvertently consume them while foraging. The potential for sexbased differences in infection arise because male Daphnia typically have a smaller body size than adult females and exposure to fungal propagules increases with size (Hall et al. 2007). Thus, size-based exposure advantages could allow males to resist infection more than females (assuming equal susceptibility to fungal spores consumed between sexes).

\section{A Motivating Field Pattern and Experimental CONFIRMATION}

\section{Methods}

Field survey.-To investigate relationships between parasites and sexual reproduction, we sampled natural epidemics across a set of lakes in southwestern Indiana (Greene and Sullivan Counties, Indiana, USA). We collected weekly or fortnightly samples throughout the epidemic season (mid August through early December) from 2009 to 2015. In total, we sampled 32 lakes, some only one year, others up to seven years. From each sampling date at each lake, we collected hosts with three vertical tows of a Wisconsin net $(13 \mathrm{~cm}$ diameter, $153 \mu \mathrm{m}$ mesh; towed bottom to surface). From these samples, we estimated several key metrics. First, from $\sim 400$ Daphnia per sample, we visually diagnosed infection status, host stage, and ephippia production with a dissecting scope at 20-50× magnification (following Ebert 2005). The absence/presence of a brood chamber distinguishes juveniles and adult stages; males have a distinctive body shape and large first antennules. For each lake-year combination, we calculated seasonal maxima for frequencies of males and ephippial females. We estimated integrated infection prevalence by calculating the area under the infection prevalence curve (Van der Plank 1963). For lakes sampled multiple years, we averaged these metrics among years $( \pm \mathrm{SE})$. We correlated maximum frequency of males and infection prevalence. (Results were similar with the mean frequency of males). All analyses used Matlab (Matlab v.9.0 R2016a; Mathworks, Natick, Massachusetts, USA).

Lake mesocosms. - To establish population-level causation between parasites and shifts in allocation to sex (indexed as male frequencies), we created experimental epidemics in large (6 m deep $\times 1 \mathrm{~m}$ wide) lake-deployed mesocosms. The experiment began during the typical autumnal epidemic season to ensure that hosts and parasites experienced natural changes in temperature, food, and daylight - the associated cues known to induce the sex response. In brief, we factorially manipulated epidemics and nutrients. (Nutrients conservatively reflect ranges from the field survey). We then tracked epidemics for $40 \mathrm{~d}$ post-inoculation (for detailed methods, see Appendix S2). As in the field survey, we quantified stage-specific and overall infection prevalence. We analyzed differences in infection prevalence among males, juvenile females, and adult females with pair-wise randomization tests (10,000 iterations). To rule out crowding as a driver of male frequency (Hobaek and Larsson 1990), we estimated host density. We analyzed differences in the maximum male frequency (calculated as for the field survey) and density among treatments with generalized linear models (GLM) with binomial and lognormal errors, respectively. For both analyses, we ran saturated and reduced models and selected the best-fitting model with chi-squared or likelihood ratio tests. For all GLM models, we tested for overdispersion using $\hat{c}$, sum of the squared residuals from the fitted GLM/residual degrees of freedom (Burnham and Anderson 2002). We used the appropriate quasi-distribution if $\hat{c}>1$.

\section{Results}

Field survey.-Maximum frequencies of males and ephippial females increased with epidemic size in the field survey (Fig. 2). Each point averages years (from 1 to 7 ). Male frequency is relative to adult females: male density/ (male density + adult female density), excluding female juveniles (results were similar including juveniles). Male frequency ( $y$-axis) was higher in lakes with larger epidemics of the focal fungal parasite ( $x$-axis, where each point is the integrated prevalence of infection in lake; $r=0.43, P=0.017$, Fig. 2A). Maximum frequency of males also positively correlated with maximal frequency of ephippium-carrying females among lakes (where each point is maximal frequency, averaged over years for a given lake; $r=0.55, P=0.001$, Fig. 2B). Together, these correlations suggest that larger epidemics led to more males, and more males led to increased sexual reproduction (indexed by frequency of ephippial females).

Time series from two lakes illustrate dynamics underlying these patterns. In these examples, we see a temporal cadence of increasing infection prevalence, then male frequency and the frequency of ephippial females (proportion of males and of ephippia-carrying females relative to non-ephippial adult females; Tessier and Cáceres 2004) through the seasonal epidemics. In the lake with a small epidemic, male production began on ordinal date 290 (1 October 2011, Fig 2C) but female hosts produced no detectable ephippia before the survey ended. In the lake with a large epidemic, male production began slightly 

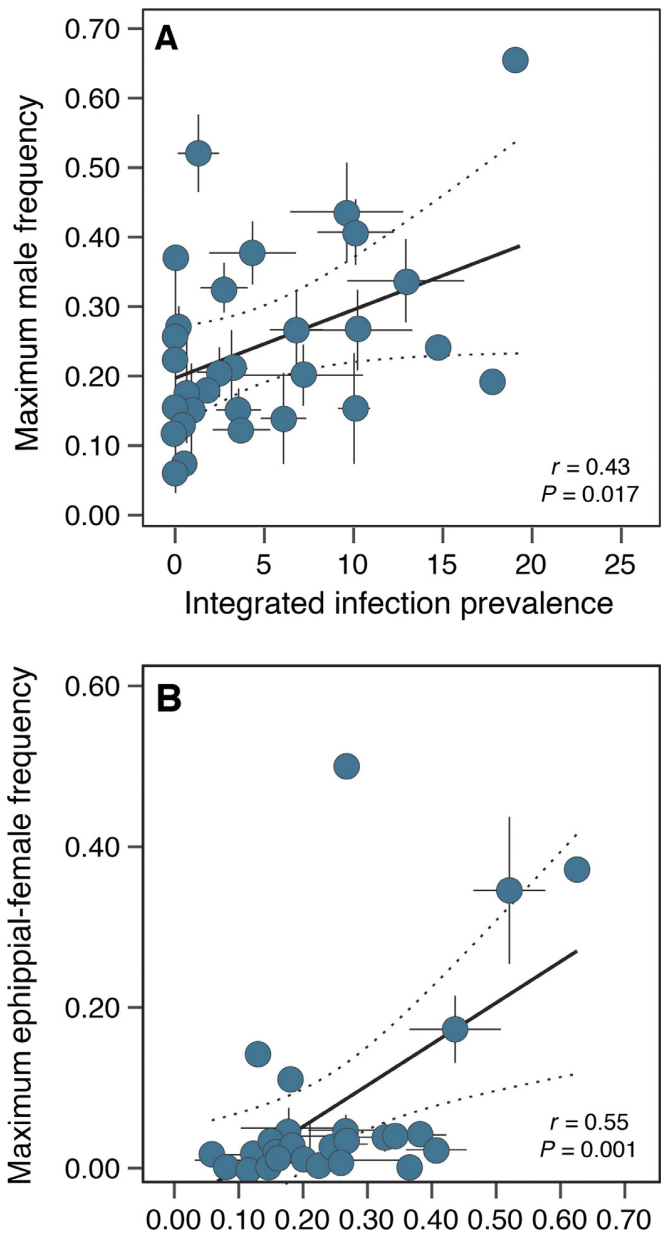

Maximum male frequency

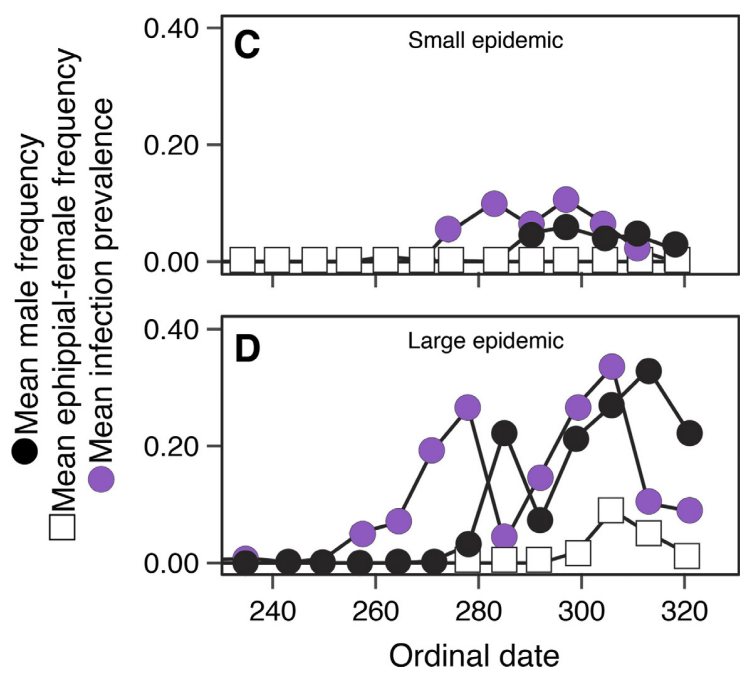

earlier, ordinal date 278 (28 September 2011, Fig 2D), and male frequency was higher relative to the small epidemic lake; ephippial production began on ordinal date 299 (26 October 2011). This chronology shows that male
FIG. 2. The epidemic-size-male-frequency-pattern field survey. Variation in the maximum frequency of males, relative to adult females, in populations of a zooplankton host with varying epidemic sizes (indexed as integrated prevalence [proportion days]). Each point is one of 32 lakes, with the maximum frequency or integrated epidemic prevalence averaged across 1-7 yrs (2009-2015). Values are mean \pm SE. Regression best-fit yrs (black line) and lower/upper 95\% confidence envelopes (gray lines) are shown. (A) Males became more frequent during larger epidemics of the fungal parasite. (B) Populations with higher maximal frequency of males had higher maximal frequency of ephippial females. (C and D) Examples showing mean frequency of infection (purple), males (black), and ephippial females (white) through the autumnal epidemic season in a lake with (C) a small fungal epidemic (Dogwood, 2011) and (D) a larger one (Midland, 2011). [Color figure can be viewed at wileyonlinelibrary.com]

and ephippial production are, in part, modulated by the onset of winter (since autumnal cooling triggers sex in this host). Yet, investment in sex was lower in the lake with a small fungal epidemic (Dogwood, 2011; Fig. 2C) relative to a lake with larger fungal epidemic (Midland, 2011; Fig. 2D). Among all lakes, similar dynamics produced the motivating correlation (Figs. 2A,B).

Lake mesocosms. - In the field experiment (Fig. 3), host populations also shifted toward higher frequencies of males during fungal epidemics, as in the field survey. Maximum male frequency increased with the addition of fungal parasites (main parasite effect $[\mathrm{P}], \chi^{2}=7.79$, $P=0.005, \hat{c}=0.07$, Fig. 3A). However, there was no effect of nutrients $\left(\mathrm{N}, \chi^{2}=0.165, P=0.685\right)$ or their interaction $\left(\chi^{2}=1.52, P=0.218\right)$. In the high nutrient treatment, infection prevalence was slightly higher (Hite et al. 2016) and male production was of longer duration (Fig. 3C,D) relative to the low nutrient treatment (Appendix S2, Fig. S1). The key point, however, is that at two varying nutrient levels, parasites increased male frequency. As expected, disease decreased host density (P, $\chi^{2}=4.61, P=0.032, \hat{c}=0.1$, Fig. 3B). However, there were no main $\left(\mathrm{N}, \chi^{2}=2.88, P=0.090\right)$ or interactive effects of nutrients on host density $(\mathrm{P} \times \mathrm{N}$, $\left.\chi^{2}=1.19, P=0.280\right)$. Thus, hosts did not produce more males due to crowding (a common stressor). In other words, crowding did not explain the epidemic-size-malefrequency pattern in the mesocosms.

Temporal dynamics in the experiment (Fig. 3C, D) largely mirror those from the field (Fig. 2C, D). They also underlie the summary patterns from the experiment (Fig. 3A). Across all high nutrient replicates, the onset of male production occurred on ordinal date 278 (5 October 2011; Fig. 3C, D). (See Appendix S2 for similar patterns in the low nutrient treatments, Fig. S1). In the absence of parasites, peak male frequency reached $\sim 52 \%$ (dashed line, both figures) on ordinal date 292 (19 October); then it declined on ordinal date 295 (22 October). With parasites, male frequency peaked later and was higher (Fig. 3D). Note that, unlike in the field survey, the experiment ended before ephippium-carrying females appeared. 

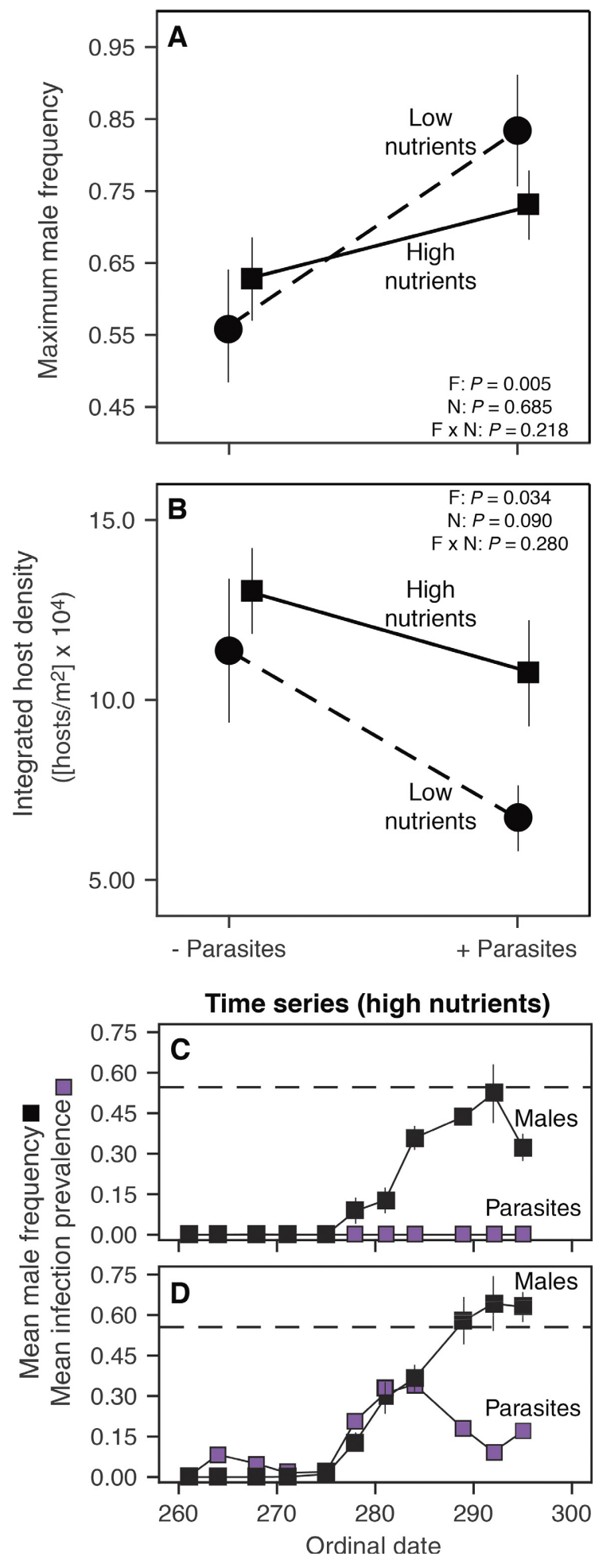

Test of the Allocation to Sex Mechanism

\section{Life table assay}

Methods.-We used a life table experiment to test for increased allocation to sex (male frequency) by
FIG. 3. Experimental confirmation of the epidemic-sizemale-frequency pattern. (A) Lake-deployed mesocosms confirmed that fungal epidemics caused host populations to shift toward higher mean frequency of males (accounting for a nutrient effect and interaction: see Results). (B) Disease significantly decreased host density. Thus, stressful overcrowding did not explain higher male frequency in parasite treatments. For GLM-produced $P$ values, $\mathrm{F}, \mathrm{N}$, and $\mathrm{F} \times \mathrm{N}$ indicate fungal parasite, nutrient, and interactive effects, respectively. (C, D) Seasonal dynamics from the high nutrient treatment illustrate mean frequency of males (black) without (-, panel C) and with (+, panel D) parasites. Purple squares denote parasite prevalence. The dashed line marks maximum frequency of males in the parasite-free treatment. Points and error bars show means \pm SE.

individual, infected females. In short, we first created six environments (flasks) that contained the requisite cues to catalyze a transition to the sexual stage (higher density, end-of-epidemic-season temperature and light conditions: $15^{\circ} \mathrm{C}, 8: 16$ light : dark cycle [Tessier and Cáceres 2004]). We added parasites to three flasks and kept the other three flasks parasite-free. After epidemics began, we collected 15 individual females from each flask and tracked their allocation to sex (number of males/total offspring produced) over three clutches while keeping them exposed to environmental cues from their natal flask (for expanded details see Appendix S2). To test for increased allocation to sex (frequency of males) and fecundity declines due to infection, we fit a mixed-effects generalized linear model (GLMM) with binomial errors (male frequency) or Poisson errors (fecundity). We checked for overdispersion with visual diagnostics and the scale parameter (Pinheiro and Bates 2000). This model also accounted for potential differences among flasks.

Results. - Data from the life table assay supported the "allocation to sex" mechanism. Infected female hosts in the life table assay significantly increased allocation to sex compared to uninfected females. These females came from and received cues from flasks where final infection prevalence $(\hat{c}=0.04, P=0.530)$ and final host density were similar across all treatments (flask $P=0.768$; spore level $P=0.433$ ). All females originally exposed in those flasks, then used for the life table assay, became infected. These infected females in the life table produced higher frequencies of males (GLMM, parasite treatment, $0.75>\hat{c}<1.4$, $\chi^{2}=5.46, P=0.019$, Fig. $\left.4 \mathrm{~A}\right)$ and produced smaller clutches $(P=0.018$, Fig. 4B). Thus, infected females incurred a parasite-mediated reduction in fecundity but allocated that reduced reproduction toward males. Hence, the epidemic-size-male-frequency pattern seen in these lakes could have arisen because infection-stressed females increased allocation to sex (male production).

\section{Development of the Male Resistance Mechanism: A Demographic Model}

The alternative male resistance mechanism poses that correlations between male frequency and epidemic size in 

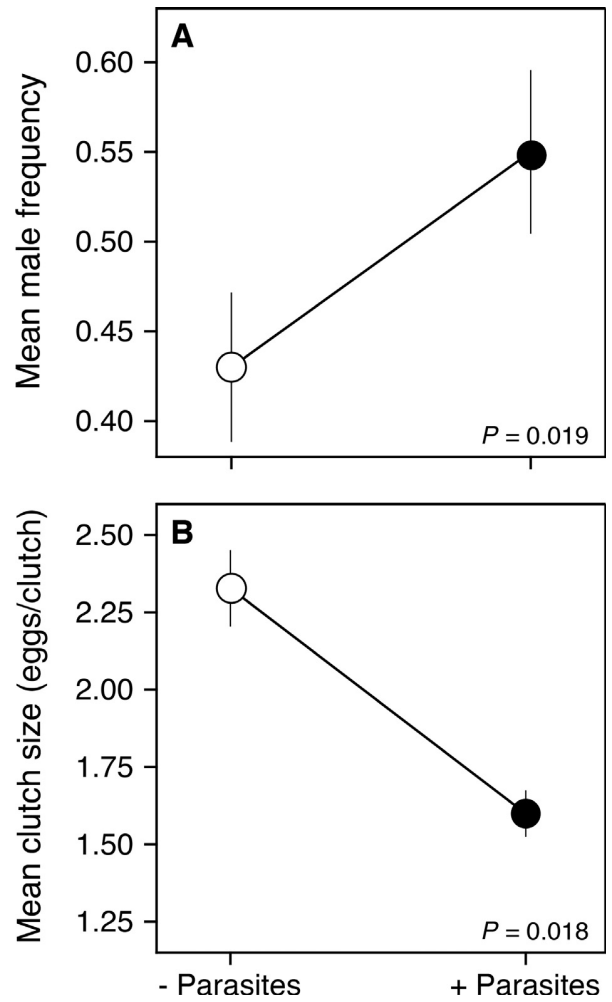

FIG. 4. Experimental test of the "allocation to sex" mechanism: life table assay. Individual, infected female hosts increase production of males in a life table assay. (A) Infected female hosts, from + parasite flasks, significantly increased the frequency of males produced per clutch. (B) Virulence on fecundity. Infected hosts produced fewer offspring relative to uninfected hosts. $P$ values come from a generalized linear mixed effects model. Filled and unfilled symbols are "- parasite" and "+ parasite" treatments, respectively. Values are means \pm SE.

the field could reflect demography. Do males resist infection and increase in frequency due to parasite-driven mortality of less-resistant females? We evaluate this possibility using a demographic model of disease, reproduction, and sexual allocation. This model separated feedbacks and identified key metrics from field and mesocosm data to evaluate the hypothesis. The details of this model appear in Appendix S1. In brief, we highly simplify reproduction, assuming that changes in male and female density reflect allocation $(s)$ to each sex from a constant reproductive flux $(R)$. Then, we assume a constant force of infection. These two assumptions removed some density-dependent feedbacks on reproduction and disease, but enabled analytical tractability. We derive conditions under which male frequency increases with larger epidemics, like in the field pattern, and over a disease-free baseline, as in the experiment. Importantly, differential mortality of infected males vs. females placed some important demographic bounds on this male-resistance mechanism. We then compared and contrasted infection prevalence of females vs. males. How does male resistance influence patterns of infection prevalence between females and males? Here in the main paper, we summarize key results from the demographic model. (For analytical and graphical details, see Appendix S1). First, the model predicts that complete male resistance (an extreme example) almost certainly leads to increasing male frequency with epidemic size (version A) and higher male frequency over a disease-free baseline (version B). However, if males become infected, moderate male resistance can (1) produce higher male frequency with epidemics and over a disease-free baseline and (2) lead to higher infection frequency in females and males. However, both infection prevalence and male frequency results depend on stage-specific mortality: males cannot suffer severe mortality from infection. This result puts some demographic bounds on the male resistance mechanism. The model readily captures the increase above the disease-free baseline version (like in the mesocosm experiment: Fig. 3). Thus, male resistance provides a mathematically viable alternative mechanism for the epidemic-size-malefrequency pattern, as long as males do not suffer extreme virulence.

\section{Quantitative Evaluation of the Alternative Mechanism: Male Resistance}

\section{Field survey and mesocosms vs. lab assay}

Methods.-We empirically tested the hypothesis that smaller males have higher resistance due to less (slower) contact with spores. First, we perform an "indirect test": we evaluated infection prevalence in the field. This test is indirect because field prevalence does not just mirror resistance. Any epidemiological model, like the one here, shows how prevalence during an epidemic combines additional traits besides resistance and various dynamical feedbacks. Therefore, prevalence can reflect resistance, assuming all else is equal. Thus, for this indirect text, we established that smaller size of males with measurements of $\sim 40$ individuals of each host stage in 23 lakes on each sampling date during epidemic season of 2015. Then, we estimated mean stage-specific infection prevalence (e.g., number of infected males/total number of males; see Appendix S2 for extended details) in each lake and mesocosm population for each sampling date.

Second, we performed a more direct test of male resistance. Specifically, we estimated resistance of each stage directly from a highly controlled lab assay, essentially eliminating the influence of other traits that also shape prevalence during field and mesocosm epidemics. In this lab experiment, we measured exposure (feeding) rate and infection prevalence (and then used those data to estimate per-spore susceptibility and resistance in the model). In brief, we measured food/spore consumption by males, juvenile females, and adult females exposed to one of three parasites doses $(0,150$, or 350 spores $/ \mathrm{mL})$ for $48 \mathrm{~h}$. We then measured hosts and maintained them for 
subsequent visual diagnosis for $19 \mathrm{~d}$ post exposure. (For details, see Appendix S2). We analyzed differences in infection prevalence from this controlled assay across stages and spore doses with logistic regression.

Results.-In the indirect test, the field survey and mesocosm experiment produced, at first glance, some support for the resistance mechanism. However, the controlled assay undermined this support. Together, these results highlight important distinctions between prevalence and resistance. In the field survey, male and juvenile female hosts were similarly sized $(P=0.175)$, but both males $(P<0.001)$ and juvenile females $(P<0.001)$ were smaller than adult females (Fig. 5A). Hence, males likely have lower exposure than adult females, all else equal (i.e., the exposure part of the hypothesis might apply). Then, in the field survey, infection prevalence was similar among males and juvenile females (squares in Fig. 5B; $P=0.409$ ) but lower than adult females (all $P<0.001$ ). The mesocosm experiment mirrored these results, except that males had lower infection prevalence relative to both female stages (high nutrient treatments, triangles in Fig. 5B, all $P<0.0001$ ); low nutrient treatments (not shown) showed similar results. However, in the controlled, lab-based assay, logistic regression quantified no difference in infection prevalence between stages, suggesting similar resistance levels among stages (for full results, with dose effects, see Appendix S2). (We discuss possible reconciliation between the indirect test [Fig. 5B] vs. the direct test estimates of infection prevalence and resistance [Figs. 5C, 6C]).

\section{A size-based model of resistance}

Methods. - In the indirect test of male resistance, field and mesocosm data suggested that males were more resistant than adult females (based on infection prevalence, which again is an indirect measure of resistance). Yet, the controlled lab experiment indicated similar infection prevalence among smaller males and larger females. Why did the size-based hypothesis for male resistance fail? To answer this question, we fit data from the lab assay to a size-based model of resistance

FIG. 5. Quantifying "male resistance": field survey and lake mesocosms vs. lab experiments. (A) In the field survey (2015), males and juvenile females were significantly smaller than adult female hosts, confirming the size component of the hypothesis. (B) In the field survey (triangles) and mesocosm experiment (squares), males and juveniles had lower infection prevalence (means \pm SE) relative to adult females. Lowercase letters indicate significant differences between stages $(P \leq 0.05)$; survey and mesocosm data analyzed separately. (C) In the lab experiment, males also tended to have lower infection prevalence relative to juvenile and adult females (means \pm bootstrapped 95\% CI). However, infection prevalence did not differ significantly across stages. $P$ values are from a logistic regression model with $\mathrm{D}$ representing parasite-dose effects, St representing stage effects, and D $\times$ St representing their interaction.[Color figure can be viewed at wileyonlinelibrary.com] (modified from Bertram et al. [2013]). For details of this model, see Appendix S2. Briefly: the model assumes that exposure, $E(L, Z)$, scales with surface area $\left(L^{2}\right)$ and with size-corrected rate $\hat{E}$ but declines with exposure to spores, $Z$ (via sensitivity $\alpha$ ). Susceptible hosts that contact spores are then infected with per spore susceptibility $u$, resistance is $\beta(L, Z)=u E(L, Z)$. We used maximum likelihood to estimate $E_{j}$ and $u_{j}$, for each host stage, $j$. We also calculated size-corrected resistance as $\hat{\beta}_{J}=\mu \hat{E}_{J}$. We then bootstrapped $95 \%$ confidence intervals around each parameter and compared estimates
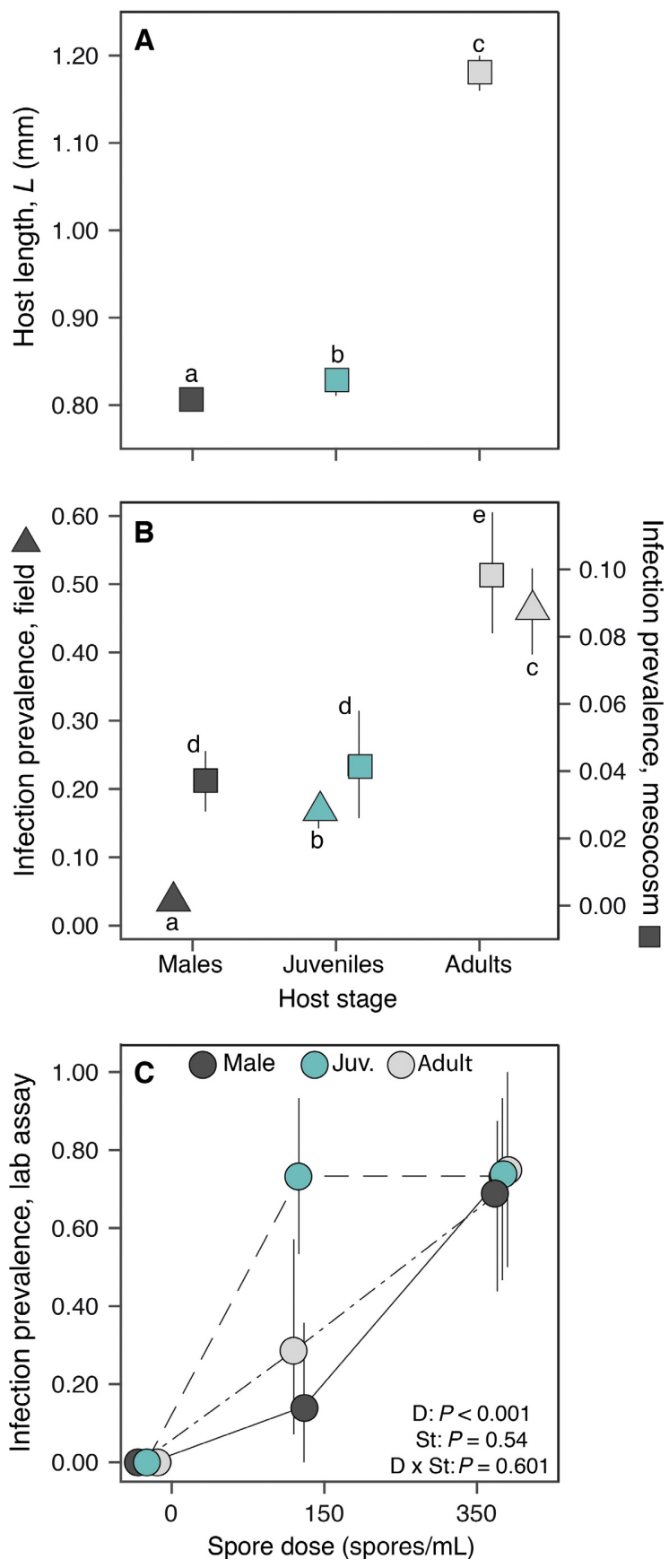

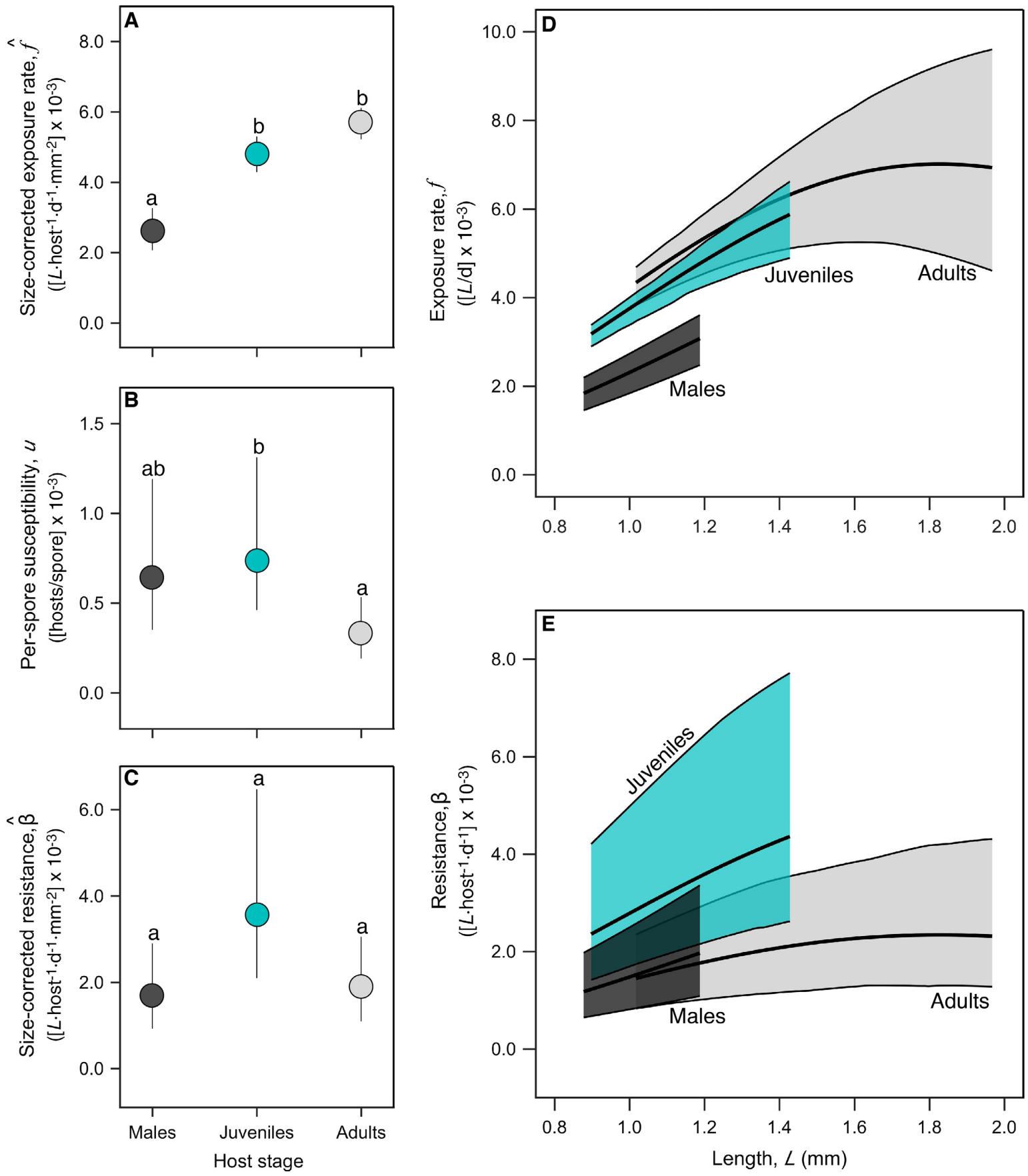

FIG. 6. Quantifying male resistance with a size-based model. A size-based model of resistance shows that smaller males do not resist infection more than adult females. (A-C) Parameter estimates (and 95\% CI) from the model (Eqs. 1 and 2) fit to a joint foraging-infection assay. (A) Size-corrected exposure rate, $\hat{E}$ (Eq. 1), (B) per-spore susceptibility, $u$ (i.e., susceptibility of hosts to infection per consumed spores), and (C) size-corrected resistance, $\beta$ (i.e., the product of $\hat{E}$ and $u$; low $\hat{\beta}$ means high resistance). (D, E) Best-fit model predictions of (D) exposure rate, $E_{j}(L, Z)$, and (E) resistance $\beta_{f}(L, Z)$, for each host stage (means and $95 \%$ confidence envelopes).Spore levels here, 350 spores $/ \mathrm{mL}$. See Appendix S2 for similar results from the assay with 150 spores $/ \mathrm{mL}$. [Color figure can be viewed at wileyonlinelibrary.com]

among stages using randomizations. Finally, we bootstrapped confidence envelopes on feeding rate, $E_{j}(L, Z)$, and resistance, $\beta_{j}(L, Z)$, as functions of length and spore dose.
Results.-The size-based model of resistance explains why males are not more resistant despite being smaller than adult females. Indeed, the size-based exposure part of the resistance model works well. In fact, compared to 
both juvenile $(P=0.0004)$ and adult females $(P=0.0004)$, males had much lower size-corrected exposure $(\hat{E})$ rates, i.e., lower foraging exposure, even after accounting for their small size (Fig. 6A). After controlling for size, juvenile and adult females had similar exposure rates (size-corrected $\hat{E} ; P=0.0684$, adults trending higher). All else being equal, then, males should have been more resistant. However, males had similar perspore susceptibility $(u)$ compared to both juveniles $(P=0.5838)$ and adult females $(P=0.1112$, Fig. 6B; adults trending lower than males) and adult females had lower per-spore susceptibility $(u)$ relative to juveniles $(P=0.0344)$. Combined, tension between exposure, $\hat{E}$, and susceptibility led to no significant differences between males and adult females in size-corrected resistance, $\hat{\beta}_{J}$, (all $P$ values of pair-wise comparisons $>0.05$, Fig. 6C). Additionally, adding in variation in size among stages, both exposure rate, $E_{j}(L, Z)$ (Fig. 6D), and resistance, $\beta_{j}(L, Z)$, increased with host size (but flattened and then decreased as large adult females depressed their feeding at high doses; Fig. 6D; see also Appendix S2 for results at lower doses, which show less foraging depression). Hence, larger adult females and smaller males had similar levels of resistance (i.e., point estimates with confidence envelopes overlapped considerably; Fig. 6E). Taken together, these results do not support the hypothesis that smaller males resist infection through lower exposure. Thus, through rigorous evaluation of male resistance, we conclude that the male resistance mechanism likely did not drive the epidemic-size-malefrequency pattern in the field.

\section{Discussion}

We evaluated two mechanisms that could link disease epidemics to the frequency of sex. In a multi-year, multilake, field survey, the frequency of males (an index of sex) was higher in lake populations of zooplankton hosts with larger fungal epidemics. A mesocosm experiment confirmed causality: the frequency of males increased with parasites relative to disease-free controls. (Since it directly manipulated parasites in the field, this experiment obviates worry about spurious correlation). Following the Abandon Ship theory (Hadany and Otto 2009), these epidemic-size-male-frequency patterns could arise if infection-stressed females increased allocation to sex (males) (Duncan and Little 2007, Mostowy and Engelstaedter 2012, Griffiths and Bonser 2013). However, it could have emerged due to population-level consequences of male resistance (a typically overlooked but important possibility that could also drive a positive relationship between epidemics and sex).

We found that individual, infected females allocated more to male offspring. Stress from infection manifested (at least in part) as virulence on fecundity; infected hosts produced clutches with fewer offspring relative to uninfected hosts. Those infection-stressed females then produced a higher proportion of males per clutch. This
Abandon Ship stress response resembles that of other facultatively pathenogenic and strictly sexual organisms that plastically alter investment in sex when stressed (e.g., by drought, low resources, and crowding; for comprehensive lists see Hadany and Otto [2009], Mostowy and Engelstädter [2012]). Here, plastic allocation choices by infection-stressed females most likely produced the sex-epidemic-size pattern seen in the field.

We arrive at that conclusion because the alternative, "male resistance," mechanism failed. Males were indeed smaller, in the field and lab experiment, than adult females. Furthermore, they had slower foraging (and thus, exposure) rates. Such size and exposure differences should have yielded male resistance. Yet, even after accounting for exposure, smaller males and larger females resisted infection similarly. The mechanistic model of resistance explained why: males were equally susceptible to infection as juvenile females and tended to be more susceptible than adult females. Furthermore, higher spore doses depressed exposure of larger adult females but not males. Both factors negated the size-based exposure advantage of males. Hence, we find no support for the male-resistance mechanism. Still, sex-based differences in resistance arise frequently in other systems (e.g., AguilarDelfin et al. 2001, Jarefors et al. 2006, Remoue et al. 2001, Walker et al. 1997) and could drive populationlevel differences in the frequency of sex more broadly.

The failure of the male resistance mechanism seemed surprising given differences in infection prevalence between males and females in the indirect test, i.e., using prevalence data from the survey and field experiment. If males resisted infection more than females, the demographic model predicted that female infection prevalence should (usually) exceed male prevalence, as seen here in the survey and experiment in the field. Yet, the resistance model and experiment ruled out male resistance. One must remember, however, that infection prevalence in the field (and fully dynamical models) does not simply mirror resistance. Hence, the contrast between the prevalencebased indirect test vs. the actual resistance metric highlights key differences between prevalence and resistance.

This allocation response by infected females did not arise due to a Red Queen mechanism. The epidemicsize-male-frequency correlation detected here superficially resembled predictions from the Red-Queen hypothesis $(\mathrm{RQH})$. In the RQH, parasites can increase frequency of sexually reproducing hosts by sometimes granting them fitness advantages over asexual hosts (via host-parasite coevolution). The RQ selection mechanism can produce positive correlations between epidemic size and frequency of sex, often indexed as percent males (Hamilton et al. 1990, Decaestecker et al. 2007, Jokela et al. 2009). However, the Daphnia-fungus system here clearly lacks essential components required for the RQH (summarized in Appendix S2). Thus, while the Red Queen provides a powerful model for parasite-induced sex, the focal system lacks most of the requisite natural history ingredients. 
Links between parasite-induced stress and allocation to sex are particularly intriguing for facultative parthenogens. For these organisms, sex intricately links to dormancy and dispersal (Bonner 1958, Bell 1982). Hence, ecological conditions that induce allocation to sex can also modulate population genetic variance, rates of evolution (Wright 1931, Balloux et al. 2003), and inbreeding depression (Cáceres et al. 2009). Therefore, connections between parasites and allocation to sex in these (and other) organisms may help clarify how and when parasites drive/maintain variation in their host populations. How generally, then, do parasites stress hosts enough to alter allocation to sex at the individual and population levels? What genetic components (e.g., modifier genes [Hadany and Otto 2009]) regulate the switch to sexual reproduction? Future studies that address these physiological and genetic questions will advance our understanding of the factors driving variation in the frequency of sexual reproduction.

\section{ACKNOWLEDGMENTS}

We are grateful to K. Boatman, A. Bowling, and Z. Brown for field assistance. S. Siscoe, R. Ronk, B. Feaster, and T. Stoelting at the Indiana DNR facilitated the field survey. Discussions with A. de Roos (UvA) and the Bever and Lively labs (IU) improved this manuscript. An EPA STAR Fellowship supported J. L. Hite. NSF GRFs supported R. M. Penczykowski, M. S. Schocket, and A. T. Strauss. NSF funded this work (DEB-0841679, 0841817, 1120316, 1120804, 1353749, 1354407, 1353806). Parameter estimates utilized Karst, funded through the Lilly Endowment and the Indiana METACyt Initiative.

\section{Literature Cited}

Aguilar-Delfin, I., M. J. Homer, P. J. Wettstein, and D. H. Persing. 2001. Innate resistance to Babesia infection is influenced by genetic background and gender. Infection and Immunity 69:7955-7958.

Balloux, F., L. Lehmann, and T. de Meeus. 2003. The population genetics of clonal and partially clonal diploids. Genetics 164:1635-1644.

Bell, G. 1982. The masterpiece of nature: The evolution of genetics of sexuality. University of California Press, Berkeley, California, USA.

Bertram, C. R., M. Pinkowski, S. R. Hall, M. A. Duffy, and C. E. Cáceres. 2013. Trait-mediated indirect effects, predators, and disease: test of a size-based model. Oecologia 173:1023-1032.

Bonner, J. T. 1958. The relation of spore formation to recombination. American Naturalist 92:193-200.

Burnham, K. P., and D. R. Anderson. 2002. Model selection and multimodel inference: a practical information-theoretic approach. Springer-Verlag, New York, New York, USA.

Cáceres, C. E., C. Hartway, and K. A. Paczolt. 2009. Inbreeding depression varies with investment in sex in a facultative parthenogen. Evolution 63:2474-2480.

Clay, K., and P. X. Kover. 1996. The Red Queen Hypothesis and plant/pathogen interactions. Annual Review of Phytopathology 34:29-50.

Cousineau, S. V., and S. Alizon. 2014. Parasite evolution in response to sex-based host heterogeneity in resistance and tolerance. Journal of Evolutionary Biology 27:2753-2766.

Decaestecker, E., A. Vergote, D. Ebert, and L. D. Meester. 2003. Evidence for strong host clone-parasite species interactions in the Daphnia microparasite system. Evolution 57:784-792.

Decaestecker, E., S. Gaba, J. A. M. Raeymaekers, R. Stoks, L. Van Kerckhoven, D. Ebert, and L. De Meester. 2007. Host-parasite 'Red Queen' dynamics archived in pond sediment. Nature 450:870-873.

Duncan, A. B., and T. J. Little. 2007. Parasite-driven genetic change in a natural population of Daphnia. Evolution 61:796-803.

Duncan, A. B., S. E. Mitchell, and T. J. Little. 2006. Parasitemediated selection and the role of sex and diapause in Daphnia. Journal of Evolutionary Biology 19:1183-1189.

Duncan, A. B., S. A. Hall, and T. J. Little. 2009. Parasitism and environmental sex determination in Daphnia. Evolutionary Ecology Research 11:965-973.

Ebert, D. 2005. Ecology, Epidemiology, and Evolution of Parasitism in Daphnia. National Library of Medicine, National Center for Biotechnology Information, Bethesda, Maryland, USA.

Ebert, D. 2008. Host-parasite coevolution: insights from the Daphnia-parasite model system. Current Opinion in Microbiology 11:290-301.

Griffiths, J. G., and S. P. Bonser. 2013. Is sex advantageous in adverse environments? A test of the Abandon-Ship hypothesis. American Naturalist 182:718-725.

Hadany, L., and S. P. Otto. 2009. Condition-dependent sex and the rate of adaption. American Naturalist 174:S71-S78.

Hall, S. R., C. R. Becker, J. L. Simonis, M. A. Duffy, A. J. Tessier, and C. E. Cáceres. 2009. Friendly competition: evidence for a dilution effect among competitors in a planktonic hostparasite system. Ecology 90:791-801.

Hall, S. R., L. Sivars-Becker, C. Becker, M. A. Duffy, A. J. Tessier, and C. E. Caceres. 2007. Eating yourself sick: transmission of disease as a function of foraging ecology. Ecology Letters 10:207-218.

Hamilton, W. D., R. Axelrod, and R. Tanese. 1990. Sexual reproduction as an adaptation to resist parasites (a review). Proceedings of the National Academy of Sciences USA 87: 3566-3573.

Hartfield, M., and P. D. Keightley. 2012. Current hypotheses for the evolution of sex and recombination. Integrative Zoology 7:192-209.

Hite, J. L., R. M. Penczykowski, M. S. Shocket, A. T. Strauss, P. A. Orlando, M. A. Duffy, C. E. Cáceres, et al. 2016. Parasites destabilize host populations by shifting stage-structured interactions. Ecology 97:439-449.

Hobaek, A., and P. Larsson. 1990. Sex determination in Daphnia magna. Ecology 71:2255-2268.

Jarefors, S., L. Bennet, E. You, P. Forsberg, C. Ekerfelt, J. Berglund, and J. Ernerudh. 2006. Lyme borreliosis reinfection: might it be explained by a gender difference in immune response? Immunology 118:224-232.

Jokela, J., M. F. Dybdahl, and C. M. Lively. 2009. The maintenance of sex, clonal dynamics, and host-parasite coevolution in a mixed population of sexual and asexual snails. American Naturalist 174:S43-S53.

Kovalchuk, I., O. Kovalchuk, V. Kalck, V. Boyko, J. Filkowski, M. Heinlein, and B. Hohn. 2003. Pathogen-induced systemic plant signal triggers DNA rearrangements. Nature 423: 760-762.

Kumpulainen, T., A. Grapputo, and J. Mappes. 2004. Parasites and sexual reproduction in psychid moths. Evolution 58:1511-1520.

Lucht, J. M., B. Mauch-Mani, H. Y. Steiner, J. P. Metraux, J. Ryals, and B. Hohn. 2002. Pathogen stress increases somatic recombination frequency in Arabidopsis. Nature Genetics 30:311-314. 
Matlab. R2016a. Mathworks, Natick, MA, USA.

Maynard Smith, J. 1978. The evolution of sex. Cambridge University Press, Cambridge, UK.

Moore, S. L., and K. Wilson. 2002. Parasites as a viability cost of sexual selection in natural populations of mammals. Science 297:2015-2018.

Mostowy, R., and J. Engelstädter. 2012. Host-parasite coevolution induces selection for condition-dependent sex. Journal of Evolutionary Biology 25:2033-2046.

O'Connell, L. M., and C. G. Eckert. 2001. Differentiation in reproductive strategy between sexual and asexual populations of Antennaria parlinii (Asteraceae). Evolutionary Ecology Research 3:311-330.

Otto, S. P. 2009. The evolutionary enigma of sex. American Naturalist 174:S1-S14.

Perkins, S. E., I. M. Cattadori, V. Tagliapietra, A. P. Rizzoli, and P. J. Hudson. 2003. Empirical evidence for key hosts in persistence of a tick-borne disease. International Journal for Parasitology 33:909-917.

Pinheiro, J. C., and D. M. Bates. 2000. Mixed effects models in $\mathrm{S}$ and S-plus. Springer New York, New York, New York, USA.
Remoue, F., D. T. Van, A. M. Schacht, M. Picquet, O. Garraud, J. Vercruysse, and A. Ly. 2001. Gender-dependent specific immune response during chronic human Schistosomiasis haematobia. Clinical and Experimental Immunology 124:62-68.

Salathé, M., R. D. Kouyos, R. R. Regoes, and S. Bonhoeffer. 2008. Rapid parasite adaptation drives selection for high recombination rates. Evolution 62:295-300.

Stelzer, C. P. 2015. Does the avoidance of sexual costs increase fitness in asexual invaders? Proceedings of the National Academy of Sciences USA 112:8851-8858.

Tessier, A. J., and C. E. Cáceres. 2004. Differentiation in sex investment by clones and populations of Daphnia. Ecology Letters 7:695-703.

Van der Plank, J. E. 1963. Plant disease: Epidemics and control. Academic Press, New York, New York, USA.

Walker, W., C. W. Roberts, D. J. P. Ferguson, H. Jebbari, and J. Alexander. 1997. Innate immunity to Toxoplasma gondii is influenced by gender and is associated with differences in interleukin-12 and gamma interferon production. Infection and Immunity 65:1119-1121.

Wright, S. 1931. Evolution in Mendelian populations. Genetics 16:0097-0159.

\section{SUPPORTING INFORMATION}

Additional supporting information may be found in the online version of this article at http://onlinelibrary.wiley.com/doi/ 10.1002/ecy.1976/suppinfo 